\title{
Factores psicosociales implicados en la actitud hacia la participación del futuro profesorado en Madrid
}

\author{
Psychosocial variables involved in the attitude toward \\ the participation of future teachers in Madrid
}

\author{
Miguel Ángel Albalá Genol ${ }^{1}$, Antonio Maldonado Rico²
}

\begin{abstract}
RESUMEN
A partir del Modelo Tridimensional de Justicia Social de Fraser (2008), compuesto por las dimensiones "Redistribución o Justicia Económica", "Reconocimiento o Justicia Cultural” y "Participación o Justicia Política”, se llevó a cabo un estudio cuantitativo del futuro profesorado, a partir de una muestra conformada por 121 estudiantes de la Universidad Autónoma de Madrid (España). El principal objetivo fue evaluar en dicha población la actitud hacia la dimensión de Participación, así como, la posible relación con los factores psicosociales analizados: la Creencia Global en un Mundo Justo (BJW) y el Autoposicionamiento Político (PI). En cuanto a los resultados, se muestran los estadísticos descriptivos de los ítems de las escalas mediante las que se evalúan las variables descritas. Además, se muestran las relaciones negativas halladas entre la actitud hacia la participación, la BJW y el PI. De cara al futuro, puede ser clave continuar investigando en el ámbito educativo cómo determinados factores psicosociales influyen en la concepción de justicia social del profesorado. Se recomienda la realización de futuros estudios con una muestra mayor, de cara a avanzar con respecto a la generalización de los resultados presentados en este trabajo.
\end{abstract}

Palabras clave: Participación, Justicia Política, Educación, Justicia Social, Estudiantes Universitarios.

\begin{abstract}
Based on the Three-dimensional Model of Social Justice (Fraser, 2008), composed of the dimensions: "Redistribution or Economic Justice”, "Recognition or Cultural Justice" and "Participation or Political Justice", a quantitative study was carried out with a sample conformed by 121 training teacher students from the Autonomous University of Madrid (Spain). The main aim of this paper is to evaluate in these students the attitude toward the participation dimension and the possible correlation with the other psychosocial variables analysed: Global Belief in a Just World (BJW) and Political Self-Positioning (PI). Regarding the results, the descriptive statistics of the items are shown. In addition, negative correlations between the attitude toward participation, the BJW, and the PI were found. In the future, it may be key to continue researching the attitudes and psychosocial variables that are related to the perception of the reality we live in. It is recommended to carry out future studies with a larger sample in order to enhance the generalization of these findings.
\end{abstract}

Keywords: Participation, Political Justice, Education, Social Justice, University Students.
Recibido: $15 / 08 / 2018$

Aceptado: $11 / 11 / 2018$
Citar: Albalá Genol, M. Á. \& Maldonado Rico, A. (2018). Factores psicosociales implicados en la actitud hacia la participación del futuro profesorado en Madrid. Investigaciones en Psicología, 23(2), pp. 5462. doi:10.32824/investigpsicol.a23n2a5 


\section{Introducción}

Desde hace décadas, el campo de la Psicología Social estudia las diferentes formas mediante las que percibimos la realidad individual y social con la que convivimos. Así, existe un continuo cuestionamiento acerca de los hechos y comportamientos que ocurren a nuestro alrededor. Este hecho puede implicar que un sujeto elabore explicaciones acerca de eventos y comportamientos que suceden a su alrededor, tanto propios como ajenos. En ocasiones, dichas explicaciones pueden estar basadas en determinados supuestos, errores e ideas preconcebidas que no siguen las reglas lógicas del tratamiento de la información, sino que se basan en métodos heurísticos simplificadores (Zubieta \& Barreiro, 2006).

La interpretación acerca de lo que es justo e injusto en nuestro contexto social cercano, o con lo que estamos implicados a nuestro alrededor, puede conllevar también la actitud que se tiene hacia participar o no, de cara a revertir las injusticias existentes. Dicha participación, se encuentra enmarcada dentro del concepto de Justicia Social que podemos denominar como Modelo Tridimensional de Justicia Social (Fraser, 2008; Murillo \& Hernández-Castilla, 2014; Jacott Jiménez et al., 2014). Desde este enfoque, se ha construido una base sólida desde la que comprender qué elementos ha de contener el constructo de justicia social en general, y el de participación o justicia política, en particular. A partir de esta perspectiva, la justicia social se concibe como constituida por tres dimensiones: en primer lugar, la dimensión justicia económica o redistribución (Rawls, 1971; Sen, 2009) aparece para referirnos a una mejor distribución de los bienes, ya sean recursos materiales o culturales. En segundo lugar, la dimensión justicia cultural o reconocimiento (Fraser, 1997) se centra en el respeto social y cultural hacia todas las personas, así como en la existencia de relaciones justas en la sociedad. En tercer lugar, en relación a la dimensión que será objeto de análisis en el presente estudio, aparece la referida a justicia política o participación (Miller, 1999; Lee \& Hipólito-Delgado, 2007), que se define como la herramienta principal para la toma de decisiones que afectan a la vida de las personas, garantizando la paridad representativa. De esta manera, la paridad participativa permite, a quienes se reconocen como integrantes de una sociedad, participar y/o disentir de la misma, partiendo de la idea básica de construir un espacio social abierto y heterogéneo que permita el debate sobre las reivindicaciones sin que la igualdad social quede en suspenso (Avendaño, 2010).

Entre las tres dimensiones descritas, la participación es la dimensión que permite articular la defensa y el mantenimiento de la justicia social en su conjunto. Podríamos decir que la dimensión de participación es la que integra y conecta las otras dos dimensiones, pues para poder formular reivindicaciones a favor de una redistribución y un reconocimiento adecuados es necesaria la promoción de la participación de los individuos afectados por las injusticias (Sainz López, 2017). Fraser (2008) plantea que las carencias en participación igualitaria derivan en injusticias en las otras dos dimensiones, ya que se niega a los individuos que no participan la posibilidad de defender sus propios intereses. En definitiva, la participación es una de las principales herramientas para revertir ciertas injusticias, y su promoción en el campo educativo puede suponer un mecanismo clave para la consecución de sociedades más justas en el futuro. En este mismo sentido, desde la perspectiva de educación para la justicia social (Murillo, Hernández-Castilla, Hidalgo \& Martínez-Garrido, 2014), es necesaria una educación que empodere al alumnado hacia el convencimiento de la participación para intervenir en su entorno, transformándolo en un lugar más justo.

Trabajos anteriores (Barreiro \& Castorina, 2015) han investigado la relación entre la representación de justicia social y diversos factores psicosociales, como la Creencia Global en un Mundo Justo (Global Belief in a Just World, en adelante BJW). La BJW fue abordada en diversos estudios llevados a cabo por Lerner $(1965 ; 1975$; 1980) y refiere a suposiciones más o menos articuladas que subyacen en la forma en que las personas se orientan hacia su entorno. Según la "hipótesis del mundo justo" (Lerner, 1975), los individuos perciben el contexto social como una relación causal en la que se recibe lo que se merece. A partir de dicha hipótesis, las personas tienen la necesidad de creer que viven en un mundo donde la gente generalmente obtiene lo que se merece, manteniendo así la sensación de control sobre el medio (Lerner \& Miller, 1978). Estas suposiciones tienen un componente funcional que está vinculado a la imagen de un mundo manejable, predecible y menos inseguro. Posiblemente, hay personas que funcionan, al menos en algunas áreas de sus vidas, únicamente dentro de estas perspectivas de predicción y control (Lerner, 1980). Así, varios autores clásicos en psicología social como como Festinger (1957), Homans (1961) o Rokeach (1971), que han tratado de comprender el comportamiento social, también han reconocido esta importante percepción de control relacionada con la BJW.

En los últimos años, también varios estudios han evidenciado, más en profundidad, hallazgos acerca de la posible relación de BJW y diversos factores sociopolíticos (Furnham, 2003) y psicosociales (Barreiro, Etchezahar \& Prado-Gascó, 2014; Zubieta \& Barreiro, 2006). Harper y Manasse (1992), analizaron las relaciones entre BJW y las actitudes hacia la pobreza. En este sentido, encontraron que la muestra participante con bajos índices en BJW mostraba más acuerdo, que la muestra con altos índices de BJW, con la afirmación: "La pobreza en el tercer mundo es debida a la explotación, guerra y sistemas económicos mundiales". Este mayor grado de acuerdo con dicha afirmación, muestra que los individuos con baja BJW manifiestan mayor acuerdo con argumentaciones alejadas de la causalidad y culpabilización para explicar la existencia de la pobreza. De esta manera, se observó un patrón de pensamiento diferente al que habían encontrado anteriormente en otro estudio Harper, Wagstaff, Newton y Harrison (1990), que observaron relación entre individuos con alta BJW y una actitud de culpabilización 
hacia las personas pobres por encontrarse en dicha situación. Por lo tanto, los hechos que ocurren en la vida cotidiana, así como las injusticias existentes que nos rodean, pueden ser percibidas e interpretadas de múltiples formas, y con ello, las actitudes y comportamientos que se generan (o se dejan de generar) hacia las mismas, de cara a poder revertirlas.

Continuando con la posible interacción de BJW con otros factores sociopolíticos, desde la Psicología Política también se recoge una distinción entre las posturas político-ideológicas y la tendencia a justificar o rechazar las desigualdades (Rottenbacher de Rojas \& Guzmán, 2013). Por un lado, por parte de las posiciones conservadoras, existe una tendencia a justificar las desigualdades existentes. Por otro lado, en relación a las posiciones progresistas se hace explícito el rechazo de estas mismas desigualdades y su deseo de eliminación. De hecho, Bobbio (1996) afirma que el criterio más frecuentemente adoptado para distinguir en política a la derecha de la izquierda es el de la diferente actitud que se asume desde cada posición frente al ideal de la igualdad, es decir, la creencia general que se tiene acerca de la posibilidad de alcanzar o no la igualdad entre las personas en la sociedad. En esta línea, se han identificado asociaciones entre la BJW y el autoposicionamiento ideológico-político, particularmente con el extremo de derechas (Barreiro et al., 2014), que pueden estar influyendo en la construcción que un individuo realiza acerca del mundo social que lo rodea, y especialmente, acerca del origen (y posibilidad o no de resolución) de las desigualdades existentes. En definitiva, se entiende el autoposicionamiento político como la vinculación entre el binomio izquierda-derecha y la identificación política, que puede observarse como un conjunto más o menos coherente de creencias, valores y actitudes sobre el sistema social y político, y el rol que la persona desarrolla en cuanto ciudadana (Costa, Etchezahar \& Melita, 2011).

Otra propuesta teórica que engloba algunos de los objetos de estudio en la presente investigación es la "teoría de la justificación del sistema" (Jost \& Banaji, 1994; Jost, Banaji, \& Nosek, 2004). Desde esta propuesta, se sostiene que las personas tienden a justificar los sistemas económicos, sociales y políticos en los que se vive, siendo con ello percibidos como justos y legítimos. Tanto la BJW, como el conservadurismo político en general, se encuentran entre las variables psicosociales y sociopolíticas que Jost \& Hunyady (2005) proponen como forma de expresión de la justificación del sistema. Además, continuando con esta corriente teórica, no solo serían los grupos más favorecidos los que tenderían a justificar la desigualdad, sino también los grupos más perjudicados desde un punto de vista económico, social, legal o político (Jost \& Hunyady, 2005). El hecho de justificar el sistema con el que convivimos es probable que pueda estar relacionado con la actitud que se tiene hacia la participación en justicia social (Rottenbacher de Rojas \& Córdova Cáceres, 2014).

Una vez conocidos algunos de los factores que podrían incidir en la perpetuación y justificación de las desigual- dades e injusticias sociales, cobra especialmente relevancia su estudio en el campo de la educación. El profesorado transmite conocimientos y valores, directa o indirectamente, relacionados con el mundo social y la interpretación de lo que ocurre en éste. Investigar diversos factores psicosociales en futuros profesores aún en formación, puede arrojar luz acerca de las diferentes formas de pensamiento que poseen en esta etapa. Además, las creencias y percepciones del futuro profesorado acerca de la posibilidad o no de revertir desigualdades existentes, podrían determinar en cierta medida algún día las creencias de su futuro alumnado en este mismo sentido. De hecho, uno de los elementos fundamentales para que el campo educativo contribuya a la creación de una mejor sociedad es que existan figuras docentes críticas, comprometidas con el alumnado y con la sociedad, sensibles ante las injusticias e implicadas en la lucha contra ellas (Smyth, 2011).

El objetivo principal del presente estudio fue analizar la actitud hacia la participación o justicia política de una muestra de estudiantes de los grados en Educación de la Universidad Autónoma de Madrid. Además, se analizaron algunas variables psicosociales y sociodemográficas, como son: la BJW, el autoposicionamiento político y el tipo de educación de procedencia (previo al acceso a la universidad), así como, las posibles correlaciones existentes entre todas estas variables.

\section{Método}

\section{Participantes}

En el estudio participaron 121 estudiantes universitarios de la Facultad de Formación de Profesorado y Educación de la Universidad Autónoma de Madrid con edades comprendidas entre 21 y 33 años $(M=22.60 ; D T=2.14)$. Como era de esperar, en función de la fuerte feminización de la profesión docente, la mayor parte de la muestra estuvo compuesta por mujeres, concretamente el $89.3 \%$ $(n=108)$, y el $10.7 \%$ restante fueron hombres $(n=13)$. El 50.4\% $(n=61)$ era alumnado de $4^{\circ}$ curso del grado de maestro/a en educación infantil y el $49.6 \%$ restante $(n$ $=60$ ) era alumnado de $4^{\circ}$ curso del grado de maestro/a en educación primaria. En relación al origen de los estudios cursados anteriormente al acceso a la Universidad, se contemplaron cinco posibles categorías: el $61.2 \%$ del alumnado participante $(n=74)$ había estudiado anteriormente en centros educativos públicos, el $24 \%$ ( $n$ = 29) procedía de una educación basada en centros concertados religiosos, seguido de, un $6.6 \%$ del alumnado que procedía de centros educativos privado-religiosos y un mismo porcentaje de alumnos con procedencia de centros concertados no religiosos. Por último, un $1.7 \%$ del alumnado $(n=2)$ era procedente de centros educativos privados no religiosos. Los sujetos participaron voluntaria y anónimamente tras brindar su consentimiento. Por todo ello, podemos afirmar que la muestra de participantes se puede considerar isomórfica respecto a la población de maestras y maestros en formación, tanto en lo relativo 
a su género declarado como respecto a sus estudios y orientación previos a sus estudios universitarios.

\section{Instrumentos}

Se administraron las siguientes pruebas:

1. Una versión adaptada de un conjunto de ítems sobre justicia social (redistribución, reconocimiento y participación) formada en este caso por cinco ítems tipo Likert, ya sometidos anteriormente a un proceso de validación en el mismo contexto de estudio en Madrid (Etchezahar, Barreiro, Jacott Jiménez \& Maldonado, 2016) y relacionados exclusivamente con la dimensión participación. Dichos ítems (tabla 1) evalúan tanto la actitud hacia la participación en el ámbito educativo, como en el ámbito político. En este sentido, en un trabajo anterior (Albalá Genol, Maldonado, Etchezahar \& Ungaretti, 2018), ya se llevó a cabo un análisis de fiabilidad de la subescala de participación, habiendo obtenido índices adecuados de fiabilidad $(\alpha=.71)$ para este conjunto especifico de ítems.

2. Una versión adaptada y validada en Argentina por Barreiro et al. (2014) de la Escala de Creencia Global en un Mundo Justo (en adelante GBJWS) (Lipkus, 1991) que ha evidenciado indicadores de fiabilidad adecuados $(\alpha=.83)$ y de validez (S-B $\chi 2 /(\mathrm{df})=2.98$; $\mathrm{CFI}=.96 ; \Delta 2=.96$; $\mathrm{RMSEA}=.08)$. Esta versión adaptada se encuentra compuesta por siete ítems (ver Tabla 2). El formato de respuesta es tipo Likert con cinco anclajes de respuesta desde 1: Totalmente en desacuerdo a 5: Totalmente de acuerdo. A mayores puntuaciones, mayores índices de BJW.

3. Una versión adaptada de la Escala de Autoposicionamiento Ideológico-político (en adelante Escala PI) de Rodríguez, Sabucedo \& Costa (1993), cuya pregunta es: "En asuntos de política la gente habla de izquierda y derecha, acorde con una escala de 1 a 10, siendo 1 de extrema izquierda y 10 de extrema derecha, ¿dónde se ubicaría usted?".

4. Variables sociodemográficas: Se diseñó un cuestionario ad-hoc para recabar este tipo de información. Entre las variables consideradas se encontraban: sexo, edad y origen de los estudios anteriores.

\section{Procedimiento}

Los sujetos fueron invitados a participar en el estudio voluntariamente, solicitando su consentimiento informado. Además, se les informó que los datos derivados de esta investigación se utilizarían solo con fines académicos y científicos. La administración de los instrumentos se realizó en una única sesión.

\section{Análisis de datos}

Para realizar el análisis estadístico de los datos se utilizó el programa SPSS 21. En primer lugar, se analizó la fiabilidad mediante "Alpha de Cronbach" (tabla 4) para la muestra de estudio de las escalas de GBJWS y partici- pación en justicia social. Posteriormente, se calcularon los estadísticos descriptivos de los ítems evaluados (media: "M" y desviación típica: "DT"), así como, los estadísticos de frecuencias en la escala PI y las variables sociodemográficas evaluadas. Por último, se llevó a cabo un análisis de correlaciones bivariadas (Pearson) para evaluar las relaciones de las puntuaciones entre la escala de participación, la escala GBJWS, la escala PI y las variables sociodemográficas evaluadas.

\section{Resultados}

En primer lugar, mediante el estadístico Alpha de Cronbach se analizó la fiabilidad para la escala de participación en justicia social $(\alpha=.73)$ obteniéndose niveles adecuados para dicha escala (Hair, Black, Babin, Anderson \& Tatham, 2006). También se calcularon los estadísticos descriptivos obtenidos a partir del conjunto de ítems que conformaron la escala empleada de actitudes hacia la participación. Como se observa en la tabla 1, en cuanto a la actitud hacia la participación de los futuros docentes evaluados las puntuaciones medias $(M)$ se encuentran entre el valor máximo $M=4.11$ (ítem 3) y mínimo $M=$ 4.67 (ítem 1) (en base a una escala de acuerdo positiva entre 1 y 5), incluyendo el grado de acuerdo con los ítems de participación relacionados tanto con el ámbito político, como con el educativo. En total, la puntuación de la actitud hacia la participación promedio hallada es $M=$ $22.59(D T=2.38)$ sobre un máximo de 25 .

Tabla 1. Estadísticos descriptivos en los ítems de la escala de participación

\begin{tabular}{lcc}
\hline Ítems escala de participación & $M$ & $D T$ \\
\hline $\begin{array}{l}\text { 1. Todos los países deberían participar en la toma } \\
\text { de decisiones internacionales que les afecten, }\end{array}$ & 4.67 & .48 \\
$\begin{array}{l}\text { aunque tengan una población pequeña. } \\
\begin{array}{l}\text { 2. Es fundamental que las/os estudiantes partici- } \\
\text { pen en la elaboración de las normas de conviven- }\end{array}\end{array}$ & 4.65 & .62 \\
cia de sus colegios. & & \\
$\begin{array}{l}\text { 3. En el Congreso debería haber representantes de } \\
\text { todas las minorías étnicas o culturales. }\end{array}$ & 4.11 & .97 \\
$\begin{array}{l}\text { 4. Las/os estudiantes deberían tener una partici- } \\
\text { pación activa sobre decisiones acerca de cómo se }\end{array}$ & 4.63 & .55 \\
gestiona el aprendizaje en las aulas. & & \\
$\begin{array}{l}\text { 5. Los gobiernos deberían convocar siempre a sus } \\
\text { ciudadanas/os cuando haya que tomar decisiones }\end{array}$ & 4.43 & .84 \\
importantes para sus vidas. & & \\
\hline
\end{tabular}

Fuente: Elaboración propia.

En segundo lugar, se obtuvo el estadístico Alpha de Cronbach para la GBJWS, hallándose niveles adecuados de fiabilidad ( $\alpha=.83$ ) en esta escala (Hair et al., 2006). También se calcularon los estadísticos descriptivos (tabla 2) de los siete ítems que componen la GBJWS (Lipkus, 1991; Barreiro et al., 2014). Las puntuaciones medias de 
los ítems se encuentran (en base a una escala de acuerdo positiva entre 1 y 5) entre el valor mínimo 1,55 (ítem 7) y máximo 2,40 (ítem 2).

Tabla 2. Estadísticos descriptivos en la escala adaptada de GBJWS

\begin{tabular}{|c|c|c|}
\hline Ítems Escala adaptada de GBJWS & $M$ & $D T$ \\
\hline $\begin{array}{l}\text { 1. Creo que las personas obtienen lo que tienen } \\
\text { derecho a tener. }\end{array}$ & 1.78 & .95 \\
\hline $\begin{array}{l}\text { 2. Creo que los esfuerzos de una persona son } \\
\text { tenidos en cuenta y recompensados. }\end{array}$ & 2.40 & .98 \\
\hline $\begin{array}{l}\text { 3. Creo que las personas se han ganado las recom- } \\
\text { pensas y castigos que reciben. }\end{array}$ & 2.17 & 1.13 \\
\hline $\begin{array}{l}\text { 4. Creo que las personas que tienen mala suerte } \\
\text { la han traído sobre sí mismas. }\end{array}$ & 1.76 & 1.06 \\
\hline 5. Creo que las personas obtienen lo que merecen. & 2.20 & 1.12 \\
\hline $\begin{array}{l}\text { 6. Creo que las recompensas y los castigos son } \\
\text { administrados justamente. }\end{array}$ & 1.69 & .89 \\
\hline $\begin{array}{l}\text { 7. Básicamente pienso que el mundo es un lugar } \\
\text { justo. }\end{array}$ & 1.55 & .79 \\
\hline
\end{tabular}

Fuente: Elaboración propia.

En tercer lugar, en relación a la evaluación mediante la adaptación de la Escala de Autoposicionamiento Político de Rodríguez et al. (1993), como se puede ver en la tabla 3, la puntuación media $(M)$ de la orientación política de los futuros docentes evaluados es de 4.31, siendo 1 el valor más cercano a la izquierda política y 10 el valor más cercano a la derecha política.

Tabla 3. Estadísticos descriptivos en la Escala adaptada de Autoposicionamiento Político

\begin{tabular}{llll}
\hline $\begin{array}{l}\text { Item Escala adaptada de Autoposicionamiento } \\
\text { Político }\end{array}$ & $M$ & $D T$ \\
\hline $\begin{array}{l}\text { Cuando se habla de política se utilizan normal- } \\
\text { mente las expresiones Izquierda y Derecha. A }\end{array}$ & & 2.31 \\
$\begin{array}{l}\text { continuación, hay una serie de casillas que van } \\
\text { de Izquierda (1) a Derecha (10), ¿En qué casilla } \\
\text { te situarías? }\end{array}$ & & \\
\end{tabular}

Fuente: Elaboración propia.

En este sentido, el $50 \%$ de los estudiantes en educación primaria e infantil evaluados $(n=60)$ se autoposicionaron dentro del rango de puntuaciones entre 1 y 4, más cercanos a la izquierda. El $25 \%$ de los participantes $(n=30)$, se autoposicionó en la puntuación 5. Finalmente, el $25 \%$ restante del alumnado participante $(n=30)$, se autoposicionó entre las puntuaciones 6 y 10, más cercanas a la derecha.

Finalmente, como se puede observar en la tabla 4 , se presentan los resultados relacionados con las relaciones existentes entre la participación del alumnado y las variables psicosociales (BJW y PI) y sociodemográficas (tipo de educación de procedencia en estudios previos a los universitarios). Por un lado, las relaciones (rc bilateral $p<.01)$ más fuertes $\left(-.39^{* *}\right)$ de carácter negativo, se hallan entre la representación de participación y la BJW de los participantes. En segundo lugar, se encuentra la relación $\left(-.34^{* *}\right)$ también en sentido inverso entre la representación de participación de los estudiantes y el autoposicionamiento político de derechas. Además, se puede observar en la tabla 4 la existencia de correlaciones entre las propias variables psicosociales y sociodemográficas evaluadas. Así, se observaron correlaciones $\left(.21^{*}\right.$ ) (rc bilateral $p<.05$ ) entre el autoposicionamiento político de derechas y la BJW del alumnado universitario evaluado. También se hallaron correlaciones (rc bilateral $p<.01$ ) inversas $\left(-.26^{* *}\right)$ entre el autoposicionamiento político de derechas y la procedencia de estudios (anterior a la universidad) relacionada con centros educativos públicos del alumnado participante del estudio.

Tabla 4. Correlaciones entre participación y variables sociodemográficas y psicosociales

\begin{tabular}{lcccc}
\hline & 1 & 2 & 3 & 4 \\
\hline Procedencia estudios & - & .11 & $.26^{* *}$ & .12 \\
BJW & & .83 & $.21^{*}$ & $-.39^{* *}$ \\
\hline PI & & & - & $-.34^{* *}$ \\
\hdashline Participación & & & & .73 \\
\hline
\end{tabular}

*. $p<.05 ; * * . p<.01$.

Nota: Alpha de Cronbach en la diagonal

Fuente: Elaboración propia.

\section{Discusión}

El objetivo principal del presente estudio era analizar las actitudes hacia la participación de una muestra de futuro profesorado de la Universidad Autónoma de Madrid, así como las posibles relaciones con algunas variables psicosociales y sociodemográficas.

En primer lugar, el conjunto de cinco ítems evaluados previamente y ya testeados en Madrid (Etchezahar et al., 2016), para evaluar la participación (como parte de la concepción tridimensional de justicia social), contribuyen a la evaluación de dicha dimensión. Las puntuaciones obtenidas, muestran en promedio altos índices de acuerdo de los futuros docentes con las cuestiones planteadas acerca de la participación tanto en la vida política, como en la escuela. No obstante, teniendo en cuenta lo novedoso que es este conjunto de ítems y de cara a poder contrastar estos resultados sería pertinente continuar testeándolos en el futuro.

En segundo lugar, en relación a los resultados procedentes de la GBJWS, como muestran los resultados descriptivos los estudiantes de educación evaluados han mostrado BJW más bajas en promedio que en otros estudios con población universitaria de psicología y sociología en Argentina (Barreiro et al., 2014), en donde la puntuación media 
fue más elevada en todos los ítems. De cara a futuros estudios en España, es necesario continuar investigando esta variable mediante la adaptación de la escala empleada, para así poder generalizar su uso con mayores garantías, ganando más representatividad si cabe.

En tercer lugar, los resultados relacionados con la escala PI (Rodríguez et al., 1993), mostraron que la mayor parte de los futuros docentes evaluados se autoposicionaron entre el centro de la escala y la izquierda política, con un promedio total de $M=4.31$. Se trata de un autoposicionamiento más hacia la izquierda política que el hallado en otros estudios similares como fue el caso de Petit, Costa y Melita (2012), que encontraron un promedio de $M=6.6$ en estudiantes universitarios de la Facultad de Ciencias Sociales de la Universidad Nacional de Lomas de Zamora (Argentina).

Por último, con respecto a los resultados del estudio de correlaciones (tabla 4), existen relaciones entre las actitudes en participación (como dimensión dentro del concepto de justicia social) y los factores psicosociales analizados. Por un lado, la representación de participación mantuvo una relación negativa con respecto a la BJW y la PI. Estos hallazgos, van en la línea de los encontrados en otros estudios anteriores, en los cuales en función de los diferentes niveles de la BJW de los sujetos se identificaron diferentes elementos en la representación social de la justicia (Barreiro \& Castorina, 2015). En este sentido, diversos investigadores han señalado que los factores psicosociales y sociopolíticos evaluados en este estudio tienen un papel relevante en los individuos como una forma de justificación del sistema económico y social, perpetuando las desigualdades existentes (Barreiro, 2009; Jost \& Hunyady, 2005). Esta justificación del sistema en el que vivimos y perpetuación de las desigualdades, está influyendo también de manera negativa en las actitudes hacia la participación que poseen los futuros docentes a medida que también muestran una mayor BJW. Asimismo, los PI más cercanos a la derecha correlacionan de manera negativa con las actitudes de participación evaluadas, así como, de manera positiva con la BJW de los sujetos. En cuanto a la variable restante por incluir en este análisis, "la procedencia de carácter público" (versus concertado y privado) de los estudios previos al acceso a la universidad, correlacionó de forma negativa con el PI de derechas. Con ello, existe una relación negativa entre el hecho de haber estudiado anteriormente al grado universitario en centros educativos públicos y poseer un PI más cercano a la derecha.

Como se ha podido observar, la actitud hacia la justicia social (en este caso desde la dimensión "justicia política o participación”), se relaciona en distintos sentidos con diversos factores psicosociales, sociopolíticos y sociodemográficos. Todo ello puede conllevar diferentes percepciones acerca de: la realidad en la que vivimos, si son justos o no los desajustes que se producen en ella, y en todo caso, el tipo de actitudes y conductas hacia revertir o no dichos desajustes. Inevitablemente, todo ello también se verá implicado en las escuelas, siendo los docentes los que puedan transmitir ciertos aspectos relacionados con la justicia social y los factores estudiados en la interpretación del sistema en el que nos integramos. Diversos investigadores en el campo de la educación han puesto de manifiesto que existen diferentes creencias, actitudes y representaciones acerca de la educación para la justicia social entre el profesorado y el alumnado de diferentes niveles (Juanes García, Jacott Jiménez \& Maldonado, 2017; Sainz López, 2017; Hernández-Castilla e Hidalgo, 2015). En el futuro, es necesario continuar desarrollando investigaciones que profundicen más acerca de los factores psicosociales, sociodemográficos y educativos que podrían incidir de forma decisiva en la forma de educar del profesorado aún en formación. Por tanto, la educación para la justicia social se enmarca en el ideal de que la educación en su conjunto se constituya como una herramienta esencial para la formación de todos los ciudadanos y, en consecuencia, para la construcción de sociedades más justas y equitativas (Krichesky et al., 2011).

Finalmente, sería recomendable tener en cuenta para futuros trabajos algunas limitaciones detectadas en el presente estudio. Por un lado, la muestra de participantes fue limitada y sería positivo ampliarla para futuros estudios. Así, podría ser recomendable contar con una muestra no tan feminizada, con una mayor cantidad de sujetos hombres. Con ello, podrían analizarse posibles diferencias por sexo en las variables psicosociales estudiadas. En relación a la muestra de estudio, otra limitación es que el estudio se realizó solo con alumnado de último curso de los grados de maestro/a en educación infantil y primaria. De cara a futuros estudios podría ser recomendable ampliar el análisis de las variables incluidas a otros cursos académicos de dichos grados. En este sentido, un objetivo de estudio futuro podría ser analizar la evolución de las variables psicosociales evaluadas a lo largo de los distintos niveles de formación universitaria del alumnado (primer año y último año de grado, posgrado, etcétera). Por último, también otra limitación puede haber sido incluir solo la dimensión "participación" de justicia social en el estudio. Para futuros trabajos podría ser objeto de estudio el análisis de las relaciones existentes entre las dimensiones "reconocimiento" y "redistribución" de la justicia social y el resto de variables psicosociales incluidas. 


\section{ANEXO \\ Escalas administradas en el estudio}

1. Escala de participación (Etchezahar, Barreiro, Jacott Jiménez \& Maldonado, 2016).

\begin{tabular}{|c|c|c|c|c|c|c|c|c|}
\hline $\begin{array}{c}\text { Totalmente en desacuerdo } \\
1\end{array}$ & $\begin{array}{l}\text { Algo en desacuerdo } \\
2\end{array}$ & $\begin{array}{l}\text { Ni de acuerdo ni en desacuerdo } \\
3\end{array}$ & $\begin{array}{l}\text { Algo de acuerdo } \\
\qquad 4\end{array}$ & \multicolumn{5}{|c|}{$\begin{array}{l}\text { Totalmente de acuerdo } \\
5\end{array}$} \\
\hline \multicolumn{4}{|c|}{ 1. Los países más ricos deberían transferir una parte significativa de sus recursos a los países más pobres. } & 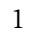 & 2 & 3 & 4 & 5 \\
\hline \multicolumn{4}{|c|}{$\begin{array}{l}\text { 2. Todos los países deberían participar en la toma de decisiones internacionales que les afecten, aunque } \\
\text { tengan una población pequeña. }\end{array}$} & 1 & 2 & 3 & 4 & 5 \\
\hline \multicolumn{4}{|c|}{$\begin{array}{l}\text { 3. Las personas con mayor riqueza deberían pagar progresivamente más impuestos que las que tienen } \\
\text { menor riqueza. }\end{array}$} & 1 & 2 & 3 & 4 & 5 \\
\hline \multicolumn{4}{|c|}{$\begin{array}{l}\text { 4. Las leyes aprobadas sin amplia mayoría en el Congreso, deberían ser ratificadas o rechazadas por el voto } \\
\text { ciudadano. }\end{array}$} & 1 & 2 & 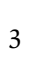 & 4 & 5 \\
\hline \multicolumn{4}{|c|}{ 5. Debería regularse por ley que los trabajadores de una empresa participen en los beneficios. } & 1 & 2 & 3 & 4 & 5 \\
\hline
\end{tabular}

2. Escala de Creencia Global en un Mundo Justo: GBJWS (Barreiro, Etchezahar \& Prado-Gasco, 2014).

\begin{tabular}{|c|c|c|c|c|c|c|c|c|}
\hline $\begin{array}{c}\text { Totalmente en desacuerdo } \\
1\end{array}$ & $\begin{array}{c}\text { Algo en desacuerdo } \\
2\end{array}$ & $\begin{array}{c}\text { Ni de acuerdo ni en desacuerdo } \\
3\end{array}$ & $\begin{array}{l}\text { Algo de acuerdo } \\
\qquad 4\end{array}$ & \multicolumn{5}{|c|}{$\begin{array}{c}\text { Totalmente de acuerdo } \\
5\end{array}$} \\
\hline \multicolumn{4}{|c|}{ 1. Creo que las personas obtienen lo que tienen derecho a tener. } & 1 & 2 & 3 & 4 & 5 \\
\hline \multicolumn{4}{|c|}{ 2. Creo que los esfuerzos de una persona son tenidos en cuenta y recompensados. } & 1 & 2 & 3 & 4 & 5 \\
\hline \multicolumn{4}{|c|}{ 3. Creo que las personas se han ganado las recompensas y castigos que reciben. } & 1 & 2 & 3 & 4 & 5 \\
\hline \multicolumn{4}{|c|}{ 4. Creo que las personas que tienen mala suerte la han traído sobre sí mismas. } & 1 & 2 & 3 & 4 & 5 \\
\hline \multicolumn{4}{|c|}{ 5. Creo que las personas obtienen lo que merecen. } & 1 & 2 & 3 & 4 & 5 \\
\hline
\end{tabular}

3. Escala adaptada de Autoposicionamiento Político: Escala PI (Rodríguez, Sabucedo \& Costa (1993).

Cuando se habla de política se utilizan normalmente las expresiones Izquierda y Derecha.

A continuación, hay una serie de casillas que van de Izquierda (1) a Derecha (10), ¿En qué casilla te situarías?

\begin{tabular}{ccccccccccc} 
Izquierda & 1 & \multicolumn{1}{l}{} & & & Derecha \\
\hline 1 & 2 & 3 & 4 & 5 & 6 & 7 & 8 & 9 & 10 \\
\hline
\end{tabular}




\section{REFERENCIAS}

Albalá Genol, M.A., Maldonado Rico, A., Etchezahar, E. \& Ungaretti, J. (2018). Social Justice and Political Orientation in Education. En E. Soriano (presidencia). VIII International Conference on Intercultural Education and I International Conference on Transcultural Health: The value of Education and Health for a transcultural and global world. Universidad de Almería, ES. Recuperado de https://www.futureacademy.org.uk/publication/EpSBS/EDUHEM2018/

Avendaño, M. (2010). La paridad participativa en la obra de Nancy Fraser. Aequalitas: Revista jurídica de igualdad de oportunidades entre mujeres y hombres, 26, 58-70.

Barreiro, A. (2009). La creencia en la justicia inmanente piagetiana: un momento en el proceso de apropiación de la creencia ideológica en un mundo justo. Psykhe, 18, 73-84. doi: 10. 4067/S0718-22282009000100007

Barreiro, A. \& Castorina, J.A. (2015). La creencia en un mundo justo como trasfondo ideológico de la representación social de la justicia. Revista Colombiana de Psicología, 24(2), 331-345.

Barreiro, A., Etchezahar, E. \& Prado-Gasco, V. (2014). Creencia global en un mundo justo: validación de la escala de Lipkus en estudiantes universitarios de la Ciudad de Buenos Aires. Interdisciplinaria, 31 (1), 57-71.

Bobbio, N. (1996). Derecha e izquierda. Razones y significados de una distinción política. Madrid, España: Taurus.

Costa, G.L., Etchezahar, E. \& Melita, G. (2011). El posicionamiento ideológico y la orientación política en jóvenes universitarios. 3er Congreso Internacional de Investigación. Universidad Nacional de La Plata, 3, 151-156. Recuperado de http://www. memoria.fahce.unlp.edu.ar/trab_eventos/ev.1538/ev.1538.pdf

Etchezahar, E., Barreiro, A., Jacott Jiménez, L. \& Maldonado Rico, A. (2016). Aportes para la evaluación de las representaciones de la Justicia Social. En C. González (Presidencia). Simposio XVI Congreso Nacional y VII Congreso Iberoamericano de Pedagogía: Democracia y Educación en el siglo XXI. Sociedad Española de Pedagogía y Universidad Complutense de Madrid. Universidad Complutense de Madrid, ES. Recuperado de https:// biblioteca.ucm.es/data/cont/docs/405-2016-10-05-LibroDeActas_SEP2016.pdf

Festinger, L. (1957). A theory of cognitive dissonance. Stanford, CA: Stanford University Press.

Furnham, A. (2003). Belief in a just world: Research progress over the past decade. Personality and Individual Differences, 34(5), 795-817. doi: 10.1016/S0191-8869(02)00072-7

Fraser, N. (1997). Justice interuptus. Critical reflextions on the "Postsocialist" Condition. Nueva York, NY: Routledge.

Fraser, N. (2008). La justicia social en la era de la política de identidad: redistribución, reconocimiento y participación. Revista de trabajo, 4(6) 83-99.

Hair, J. F., Black, W. C., Babin, B. J., Anderson, R. E. \& Tatham, R. L. (2006). Multivariate Data Analysis. New Jersey: Pearson.

Harper, D. J., Wagstaff, G. F., Newton, J. T. \& Harrison, K. R. (1990). Lay causal perceptions of third world poverty and the just world theory'. Social Behavior and Personality: an international journal, 18 (2), 235-238.
Harper, D.J. \& Manasse, P. R. (1992). The Just World and the Third World: British explanations for poverty abroad. The Journal of social psychology. 132 (6), 783-785. doi: 10.1080/00224 545.1992.9712107

Hernández-Castilla, R. \& Hidalgo Farran, N. (2015). Medición de las actitudes de los docentes hacia la justicia social (EAJSE). Instrumento y validación. En AIDIPE (Ed.), Investigar con y para la sociedad (Vol. 1, pp. 81-89). Cádiz, ES: Bubok. Recuperado de http://aidipe2015.aidipe.org

Homans, G. C. (1961). Social Behavior: Its Elementary Forms. New York, NY: Harcourt. Brace and Hovanovich.

Jacott Jiménez, L., Maldonado Rico, A., Sainz López, V., Juanes García, A., García- Vélez, T. \& Seguro, V. (2014). Representations of social justice amongst Spanish teachers and students. En P. Cunningham y N. Fretwell (Eds.), Innovative Practice and Research Trends in Identity, Citizenship and Education (pp. 122-139). Londres, UK: CiCe.

Juanes García, A., Jacott Jiménez, L. \& Maldonado Rico, A. (2017). Representaciones de Justicia Social en profesores en formación. Revista Internacional de Didáctica y Organización Educativa, 3 (2), 26-45.

Jost, J. T., \& Banaji, M. R. (1994). The role of stereotyping in system justification and the production of false consciousness. British journal of social psychology, 33 (1), 1-27. doi: 10.1111/ j.2044-8309.1994.tb01008.x

Jost, J. T., Banaji, M. R. \& Nosek B.A. (2004). A decade of system justification theory: Accumulated evidence of conscious and unconscious bolstering of the status quo. Political psychology, 25(6), 881-919.doi:10.1111/j.14679221.2004.00402.x

Jost, J. T. \& Hunyady, O. (2005). Antecedents and consequences of system-justifying ideologies. Current directions in psychological science, 14 (5), 260-265.

Krichesky, G. J., Martínez-Garrido, C., Martínez Peiret, A. M., García Barrera, A., Castro Zapata, A. \& González Bustamante, A. (2011). Hacia un Programa de Formación Docente para la Justicia Social. REICE. Revista Iberoamericana sobre Calidad, Eficacia y Cambio en Educación, 9 (4), 63-77.

Lee, C. C. \& Hipólito-Delgado, C. (2007). Counselors as agents of social justice. In C.C. Lee (Ed.). Counseling for Social Justice, (pp. 13-38). Alexandria, VA: American Counseling Association.

Lerner, M. J. (1965). Evaluation of performance as a function of performer's reward and attractiveness. Journal of Personality and Social Psychology, 1 (4), 355-360. doi: 10.1037/h0021806

Lerner, M. J. (1975). The justice motive in social behaviour: Introduction. Journal of social issues, 31 (3), 1-19.

Lerner, M. J. \& Miller, D. T. (1978). Just world research and the attribution process: Looking back and ahead. Psychological bulletin, 85 (5), 1030-1051.

Lerner, M. (1980). The belief in a just world: a fundamental delusion. New York, NY: Plenum.

Lipkus, I. (1991). The construction and preliminary validation of a global belief in a just worlds scale and the exploratory analysis of the multidimensional belief in a just world scale. Personality and Individual Differences, 12 (11), 1171-1178. doi: 10.1016/0191-8869(91)90081-L

Miller, D. (1999). Principles of Social Justice. Cambridge, MA: Harvard University Press. 
Murillo, F. J., Hernández-Castilla, R., Hidalgo, N. \& MartínezGarrido, C. (2014). Elaboración y Evaluación Psicométrica de la Escala de Actitudes hacia la Justicia Social en Educación (EAJSE). Revista Internacional de Educación para la Justicia Social (RIEJS), 3 (2), 215-233.

Murillo, F. J. \& Hernández-Castilla, R. (2014). Liderando Escuelas Justas para la Justicia Social. Revista Internacional de Educación para la Justicia Social (RIEJS), 3 (2), 3-32.

Petit, L., Costa, G. L. \& Melita, G.B. (2012). Orientación política y posicionamiento ideológico en estudiantes de la Facultad de Ciencias Sociales de la Universidad Nacional de Lomas Zamora. Hologramática, 127 (3), 139-148.

Rawls, J. A. (1971). A theory of justice. Cambridge: Harvard University Press.

Rodríguez, M., Sabucedo, J. M. \& Costa, M. (1993). Factores motivacionales y psicosociales asociados a los distintos tipos de acción política. Psicología Política, 7, 19-38.

Rokeach, M. (1971). Long-range experimental modification of values, attitudes, and behavior. American psychologist, 26 (5), 453-459.

Rottenbacher de Rojas, J. M. \& Córdova Cáceres, C. (2014). El autoritarismo y la justificación de la inequidad como condicionantes ideológicos de los niveles de deliberación y participación política. Avances en Psicología Latinoamericana, 32 (3), 495-513. doi: 10.12804/apl32.03.2014.10
Rottenbacher de Rojas, J. M. \& Guzmán, J. R. (2013). Intolerancia a la Ambigüedad, Conservadurismo Político y Justificación de la Inequidad Económica, Legal, Educativa y Étnica en la Ciudad de Lima-Perú. Revista Colombiana de Psicología, 22(2), 253-274.

Sainz López, V. (2017). Representaciones de la justicia social en profesores y estudiantes de Educación Secundaria (Tesis Doctoral, Universidad Autónoma de Madrid). Recuperada de https://repositorio.uam.es/bitstream/handle/10486/680617/ sainz_lopez_vanesa.pdf?sequence $=1$

Sen, A. (2009). The idea of Justice. Nueva York: Penguin Press. Madrid, ES: Taurus.

Smyth, J. (2011). Critical pedagogy for social justice. Nueva York, NY: Continuum.

Zubieta, E. \& Barreiro, A. (2006). Percepción social y creencia en el mundo justo. Un estudio con estudiantes argentinos. Revista de Psicología, 24 (2), 175-196. 